\title{
Introgression in the genus Campylobacter: generation and spread of mosaic alleles
}

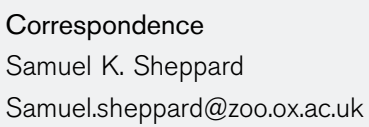

Received 2 September 2010

Revised 2 December 2010

Accepted 3 January 2011

\author{
Samuel K. Sheppard, Noel D. McCarthy, Keith A. Jolley \\ and Martin C. J. Maiden \\ Department of Zoology, University of Oxford, South Parks Road, Oxford OX1 3PS, UK
}

\section{INTRODUCTION}

The availability of multi-locus genetic data from large collections of bacterial isolates has demonstrated the central role of horizontal genetic exchange in the evolution of many bacterial populations (Didelot \& Maiden, 2010; Fraser et al., 2007). The extent of this process varies from essentially none, in clonal monomorphic bacteria such as Mycobacterium tuberculosis (Gutacker et al., 2006) and Salmonella Typhi (Kidgell et al., 2002), to extensive in Neisseria meningitidis, Streptococcus pneumoniae, Staphylococcus aureus (Feil \& Spratt, 2001) and Campylobacter jejuni (Sheppard et al., 2008; Wilson et al., 2009), and extreme in Helicobacter pylori, which has a non-clonal population structure (Falush et al., 2001). Horizontal acquisition of genes influences bacterial ecology and evolution (Ochman et al., 2000), for example by increasing the rate at which favourable mutations accumulate within a population (Baltrus et al., 2008; Cooper, 2007), or by

Abbreviations: MLST, multi-locus sequence typing; ST, sequence type.

A supplementary figure showing neighbour-joining trees for individual allele sequences is available with the online version of this paper. conferring novel metabolic capabilities (Lawrence, 1999; Spratt et al., 2001), and in recombinogenic species it can be more important than mutation in determining the genome content (Feil \& Spratt, 2001; Ochman \& Groisman, 1994).

Genetic exchange was first demonstrated in the bacteria in the 1940s (Lederberg \& Tatum, 1946), but it was with the widespread application of DNA sequencing methods at the end of the 20th century that its extent and evolutionary significance were appreciated, challenging the concept that all bacteria were essentially asexual organisms (Maynard Smith et al., 2000). Multi-locus sequence typing (MLST) (Maiden, 2006), and related techniques, demonstrated that, notwithstanding high frequencies of genetic exchange in many bacterial populations, seven-locus allelic profiles (sequence types, STs) contained sufficient information to associate genotype clusters with phenotypic properties commonly associated with microbiological species and subspecies groups (Hanage et al., 2005; Maiden, 2006; Sheppard et al., 2008).

In addition to the reshuffling of intact genes, intragenic recombination between genetically divergent loci can generate new 'mosaic' alleles. These are alleles where 
portions of the nucleotide sequence have different evolutionary histories combined in a single gene allele (Maynard Smith et al., 1991; Milkman \& Stoltzfus, 1988). Mosaic alleles, which are readily generated in bacteria because RecA-mediated DNA repair systems can accept the import of DNA with up to $30 \%$ sequence divergence (Lorenz \& Wackernagel, 1994), have been described in genera as diverse as Streptococcus (Hollingshead et al., 2000; Kapur et al., 1995), Neisseria (Maiden et al., 1996; Maynard Smith et al., 1991; Spratt, 1988; Spratt et al., 1991) and Escherichia (Milkman \& Stoltzfus, 1988; Stoltzfus et al., 1988). Many studies have focused on mosaic genes that have obvious phenotypic effects, such as increased antibiotic resistance (Coffey et al., 1995; Dowson et al., 1990), virulence (Halter et al., 1989) and antigenic variation (Snyder et al., 2007), where positive selection can be invoked to explain the spread of rare novel variants in a population; however, mosaic alleles have also been described among housekeeping genes that are subject to stabilizing selection (Dingle et al., 2005; Zhou \& Spratt, 1992).

Because the donor and recipient regions of the mosaic housekeeping genes can potentially be identified, they can provide information on the patterns of gene flow in the absence of overt strong positive selection (Brückner et al., 2004; Hakenbeck, 1998; Maynard Smith et al., 1991; Milkman \& Stoltzfus, 1988; Maynard Smith, 1992; Stoltzfus et al., 1988). Using mosaic alleles to investigate gene flow has advantages over whole-allele replacements (Hanage et al., 2005; Sheppard et al., 2008), firstly because the potential for incorrectly defined mixed STs (CaroQuintero et al., 2009) is removed, and secondly because the recombination profile representing a single genetic event can be traced through the population to determine patterns of recombination. This allows the different types of hybrid proliferation to be differentiated, including founding events, clonal expansion and within-species horizontal transfer.

Recombination, and barriers to it, have been identified as an important force in determining bacterial population structure (Hanage et al., 2006; Lawrence, 2002). However, many fundamental parameters have yet to be established and the relationship of bacterial population structure to 'species' remains a matter of debate (Cohan \& Koeppel, 2008; Doolittle, 2008; Fraser et al., 2009). Large-scale nucleotide-sequence-based population studies permit the determination of the relative rates and mechanisms of gene flow among populations that are necessary to test the various theoretical paradigms and to investigate the role of neutral and selective forces in these processes (Fraser et al., 2009). In this study, we investigated bacterial 'introgression' - the movement of DNA between recognized species - in the species Campylobacter jejuni and Campylobacter coli, which together represent the major cause of bacterial gastroenteritis in many parts of the world (Sheppard et al., 2009). Mosaic alleles among C. jejuni and C. coli genotypes were characterized and used to investigate the movement and spread of genetic material between these two closely related taxa (Friis et al., 2010), confirming the observations made on the exchange of whole MLST loci (Sheppard et al., 2008), and demonstrating the pattern of horizontal gene transfer and the fate of transferred genetic material.

\section{METHODS}

Genotype data. Campylobacter jejuni and Campylobacter coli genotype information was obtained from the publicly accessible MLST database (http://pubmlst.org/campylobacter) (Jolley et al., 2004). The C. jejuni and C. coli data archive contains ST information for seven housekeeping loci: aspA, glnA, gltA, glyA, pgm ( $g \operatorname{lm} M)$, tkt and $u n c A(\operatorname{atp} A)$, positioned $\geqslant 15 \mathrm{~kb}$ apart on the bacterial genome (Dingle et al., 2001). Alleles termed pgm and uncA in the MLST scheme have been renamed $\operatorname{glm} M$ and atpA respectively in later genome annotations, but their names have been retained within the MLST scheme, for consistency with previous studies. The alleles from 2953 distinct STs were analysed.

Identification of mosaic alleles. Candidate mosaic alleles were identified using the model-based clustering algorithm implemented in the software STRUCTURE (Pritchard et al., 2000). All the alleles were analysed for each of the seven MLST loci to identify the genetic ancestry of the allelic variants. A population number $(k)$ of 2 was used. This ensured that the analysis robustly identified putative ancestry to the two species (C. jejuni and C. coli), which are approximately $12 \%$ divergent at the nucleotide level. Alternative values for $k$, for example $k=4$ to reflect the three-clade structure within C. coli, were not used because the nucleotide identity within $C$. jejuni is $>98.5 \%$ and among the three C. coli clades it is $>93 \%$. Therefore, variation between MLST alleles within these two species is generally $<10$ polymorphisms, compared to $>50$ between species. This affects the robustness of population assignment using STRUCTURE. Alleles where the probability of belonging to either the $C$. jejuni or the $C$. coli population was $\leqslant 0.75$ were considered as possible mosaic alleles. A cut-off of 0.75 was chosen to conservatively detect potential mosaic alleles.

Recombinant fragment characterization. STRUCTURE-based analysis was used to confirm whether possible hybrids identified were mosaic alleles. This involved the characterization of potential mosaic alleles using a linkage model (Falush et al., 2003), which allowed for disequilibrium in linkage between loci, in this case nucleotides, and enabled the identification of the position of inter-specific recombination events. A comparison dataset $(n=194)$, which described the diversity of C. jejuni (122) and C. coli (72) STs in the pubMLST database (Jolley et al., 2004), was analysed alongside the STs containing mosaic alleles $(n=81)$. The population of origin (POPINFO) was assigned for all the STs in the input file, but only the non-recombinant isolates were used, as a training dataset (POPFLAG), to define the background population structure. Origin-population was assigned probabilistically for each nucleotide (3309 bp) in all of the STs including those containing mosaic alleles. 10000 burn-in cycles were run with 10000 additional repetitions for all the analysis. Otherwise, the no admixture model was used with default settings.

Clonal relationships. The genealogy of the STs was estimated using CLONALFRAME, a model-based approach to determining microevolution in bacteria (Didelot \& Falush, 2007), which calculates clonal relationships with improved accuracy as it distinguishes point mutations from imported chromosomal recombination events, which are the source of the majority of allelic polymorphisms. CLONALFRAME analysis was carried out on concatenated sequences of the same 275 STs as used in the STRUCTURE analysis (above). The program was run with a burn-in of 50000 burn-in iterations followed by 50000 data collection iterations. The consensus tree represents combined data 
from three independent runs with $75 \%$ consensus required for inference of relatedness.

Origin of allelic recombination. The origin of the largest putatively imported region within each mosaic allele was determined using the BLAST algorithm (Altschul et al., 1990). Sequences were compared to a library database of all the non-recombinant alleles at that locus and the origin was assigned based on the highest identity (\%) of the longest possible alignment region. Mosaic alleles, and the origin of recombination determined using BLAST, were indicated on phylogenies of individual alleles reconstructed using MEGA software, version 3.1, using the Kimura two-parameter model and neighbour-joining clustering (see Supplementary Fig. S1, available with the online version of this paper).

Structuring of species and gene flow. The number of fixed differences, shared polymorphisms and between-population gene flowestimated by $F_{\mathrm{ST}}$ - were calculated using the DnaSP V 4.0 (Rozas et al., 2003) and Arlequin V 3.1 (Excoffier et al., 2005) software packages. Formal species assignment of STs was carried out as previously described (Sheppard et al., 2008) using STRUCTURE, with a threshold probability of $0.75(75 \%)$ being used as the cut-off for membership of a particular ST to each species, and CLONALFRAME for clade assignment of $C$. coli haplotypes. Combining these data enabled the assignment of each ST, including those containing mosaic alleles, to a given species/clade for quantitative analysis of gene flow between groups.

Micro-evolutionary analysis of polymorphisms. Analysis was carried out to investigate the polymorphic sites within host and donor regions of mosaic alleles in the four largest mosaic allele clusters (see Fig. 3). Polymorphic sites were characterized within the recombinant and non-recombinant portions of each mosaic allele using START2 (Jolley et al., 2001) and MEGA (Kumar et al., 2004) software, and two analyses were carried out to investigate the polymorphisms within C. coli and C. jejuni alleles. First, individual polymorphisms within recombinant regions were compared to those in non-recombinant alleles and the synonymous and non-synonymous nucleic acid substitutions were quantified. Second, the Baysian analysis software STRUCTURE was used to probabilistically assign polymorphisms within recombinant regions to $C$. jejuni or $C$. coli to determine if the conserved polymorphisms were putatively more similar to those associated with one of the two species. In some cases there were low numbers of nucleotide polymorphisms within the MLST alleles; for example there were only two nucleotide differences between the mosaic alleles aspA-87 and aspA-117, which limited the extent to which inference could be made.

\section{RESULTS}

\section{Mosaic allele characterization}

From the 2953 STs examined, which contained 1738 alleles, a total of 31 alleles were defined as mosaics on the basis of population assignment probabilities of $\leqslant 0.75$ to groups corresponding to either $C$. jejuni or $C$. coli (Fig. 1). Further analysis identified putative recombination points in each mosaic which were consistent with mixed ancestry. The greatest number of mosaic alleles was recorded in the aspA locus (12 mosaic alleles) and tkt locus (11 mosaic alleles). There were three alleles defined as mosaic in the glt $A$ and glyA loci and one in the $p g m$ and $u n c A$ loci. No evidence was found for mosaic alleles at the $g \ln A$ locus. The 31 mosaic alleles were distributed among 81 STs. Sixteen mosaic alleles were specific to one ST, 11 were found in two to five STs, mosaic alleles aspA-87 and gltA-134 were each found in 8 STs, $t k t-12$ was in 11 STs and $t k t-169$ was in 12 STs.

\section{The origin of recombinant regions}

The distribution of mosaic genes was asymmetrical between $C$. jejuni and $C$. coli, with six of the mosaic alleles

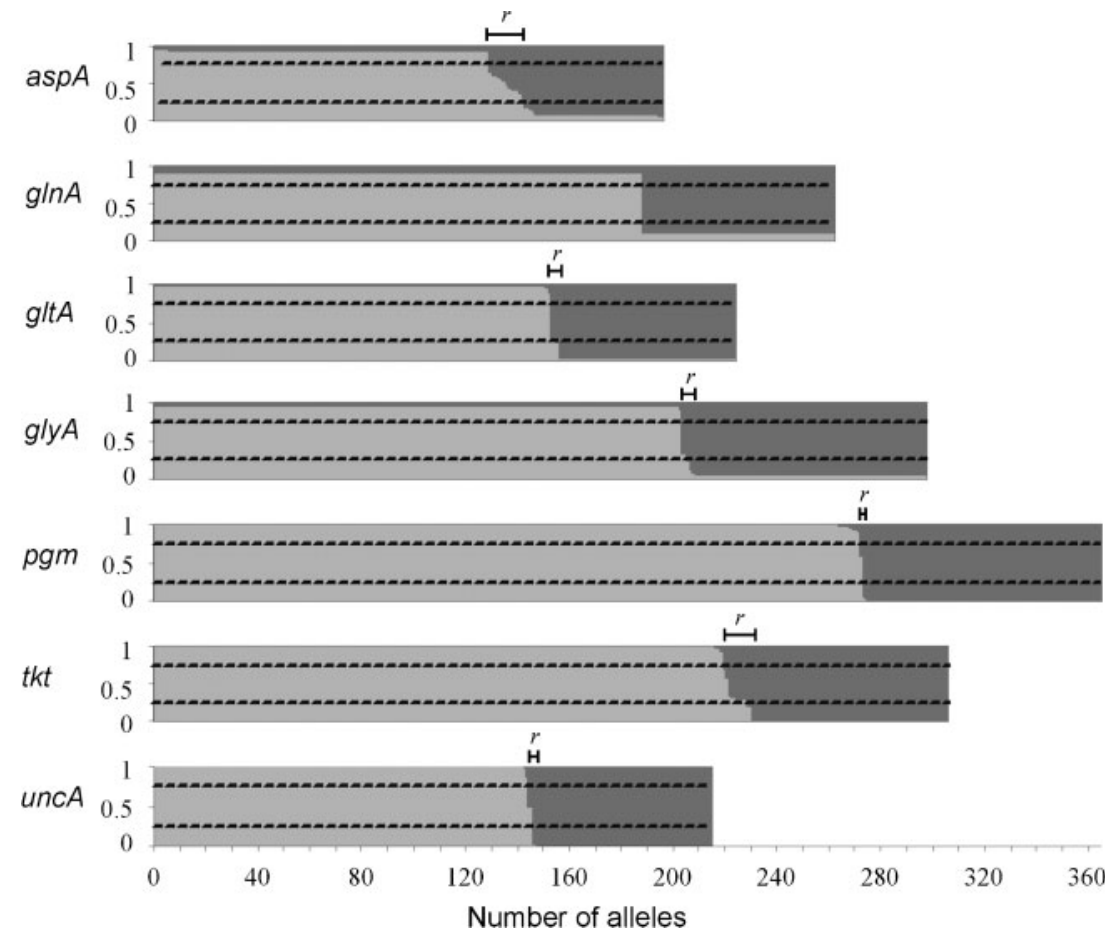

Fig. 1. Identification of mosaic alleles: cluster analysis using STRUCTURE inferring the probability-based genetic ancestry for allelic variants of the seven MLST loci (aspA-uncA). Each unique allele sequence is represented with vertical lines, divided into two shaded regions indicative of genetic ancestry to $C$. jejuni (light grey) or C. coli (dark grey). From this information inter-specific recombination between these species can be inferred. Alleles not assigned to a single genetic ancestry $(P \geqslant 0.95)$, and with assignment probability $\leqslant 0.75$, are considered inter-genomic recombinants $(r)$. The analyses were carried out with $k=2$. 
Table 1. Predicted origin of mosaic alleles given for genotypes assigned to C. jejuni and C. coli clades 1 to 3

\begin{tabular}{|lcccc|}
\hline & \multicolumn{4}{c|}{ Percentage of total number of alleles $(\boldsymbol{n})$} \\
\cline { 2 - 5 } Recombination & $\begin{array}{c}\text { C. jejuni } \\
\text { with: }\end{array}$ & $\begin{array}{c}\text { C. coli clade } \mathbf{( 1 2 4 2 )} \\
\mathbf{( 3 0 2 )}\end{array}$ & $\begin{array}{c}\text { C. coli clade 2 } \\
(\mathbf{7 4})\end{array}$ & $\begin{array}{c}\text { C. } \text { coli clade 3 } \\
(\mathbf{1 2 6})\end{array}$ \\
\hline C. jejuni & 91.72 & 8.28 & 0 & 0 \\
C. coli clade 1 & 0.40 & 99.6 & 0 & 0 \\
C. coli clade 2 & 0 & 0 & 100 & 0 \\
C. coli clade 3 & 0.08 & 0 & 0 & 99.92 \\
\hline
\end{tabular}

representing horizontal gene transfer from C. coli into $C$. jejuni STs and the remaining 25 from $C$. jejuni into C. coli STs. The direction of gene transfer, estimated by BLAST assignment of the recombinant regions, identified the ancestry of the recombinant region of the mosaic alleles (Supplementary Fig. S1). In alleles aspA-54, aspA-87, aspA120, glyA-208, glyA-240 and tkt-166, this could be assigned to an identifiable $C$. jejuni lineage. The possible origin of the recombinant regions in the other mosaic alleles was more ambiguous because there was insufficient sequence variation in the derived allele to identify a particular donor lineage. However, recombination estimates were possible at the species or clade level and all but one episode of gene flow between C. jejuni and C. coli involved a single C. coli clade (clade 1). The exception was $t k t-194$, found in a single $C$. jejuni ST, which had regions of $C$. jejuni and $C$. coli clade 3 origin.

\section{Inter-species gene flow}

Quantification of mosaic alleles allowed the investigation of patterns of gene flow. Nineteen $(0.86 \%)$ of the $2221 \mathrm{C}$. jejuni STs contained mosaic alleles and $105(16.56 \%)$ of the 634 C. coli STs contained mosaic alleles. No mosaic alleles were found among STs from C. coli clades 2 and 3. Patterns of allele distribution, quantified based on the proportion of the total number of alleles and the BLASTdefined origin of the recombinant fragment, gave a conservative estimate of gene flow (Table 1). C. coli had acquired DNA from C. jejuni in $8.3 \%$ of alleles, 17 times more prevalent than the reciprocal process $(0.5 \%$ of $C$. jejuni alleles were mosaics).

\section{Clonal structure and allele mosaics}

A CLONALFRAME genealogy, generated with concatenated MLST gene sequences, partitioned C. jejuni and C. coli into two distinct groups, which was consistent with the $F_{\mathrm{ST}}$ values of 0.86 (DnaSP) and 0.87 (Arlequin) calculated from the same data. Eleven STs containing mosaic alleles were found within C. jejuni and 70 in C. coli clade 1. The mosaic alleles were examined in relation to this genealogy (Fig. 2). Mosaicism could be indicative of non-contiguous imports, as has been observed in Helicobacter pylori (Kulick et al.,
2008), but shared patterns of mosaicism among alleles and the clustering of mosaic alleles on the tree are likely to be indicative of single founding events followed by subsequent expansion. Based on this assessment there was evidence for 13 introductions, five among $C$. jejuni and eight among $C$. coli (Fig. 2). Recombination events between C. jejuni and C. coli had varying levels of subsequent clonal expansion, indicated by clusters of related STs containing the same mosaic allele. For example, the putative introduction into C. coli, termed 'coli 1', which generated alleles aspA-87, aspA-126, aspA-120, aspA-117, aspA-157 and aspA-115, had expanded into 16 clonally related lineages (Figs 2 and 3). There was also evidence of horizontal transfer of mosaic alleles following initial founding events within both $C$. jejuni and C. coli. The mosaic pgm-93 allele was found in two distantly related $C$. jejuni lineages, and $t k t-164$ and $t k t$ 168 were both found in the two largest $C$. coli tkt mosaic allele clusters (Figs 2 and 3). In all ST clusters that contained more than one mosaic allele, the recombination pattern appeared non-random with shared terminal ends, indicative of subsequent recombination events after the acquisition of a mosaic allele. For example, in the two main clusters of $t k t$ mosaic alleles, the uniformity of the terminal position of the recombinant regions was consistent with whole-allele replacements being subsequently eroded by reacquisition of C. coli DNA (Fig. 3).

\section{Micro-evolution of mosaic alleles}

Some indication of possible genetic events in the evolution of the mosaic genes may be evident from patterns of sequence polymorphism within them. However, in this study the strength of these inferences was constrained by the limited number of polymorphisms within the mosaic alleles. The most parsimonious explanation for genetic relatedness within the four largest mosaic allele clusters (aspA, gltA, tkt1, tkt2 - Fig. 3) was a single founding recombination event followed by subsequent clonal expansion accompanied by diversification due to mutation and recombination events. Within these mosaic allele clusters (Fig. 3), the total numbers of non-synonymous $(n)$ and synonymous $(s)$ base substitutions in all the recombinant regions in each cluster were 11,171 (aspA); 2, 8 $(g l t A) ; 7,12(t k t 1)$; and 26, 49 (tkt2). The number expected 
(a)

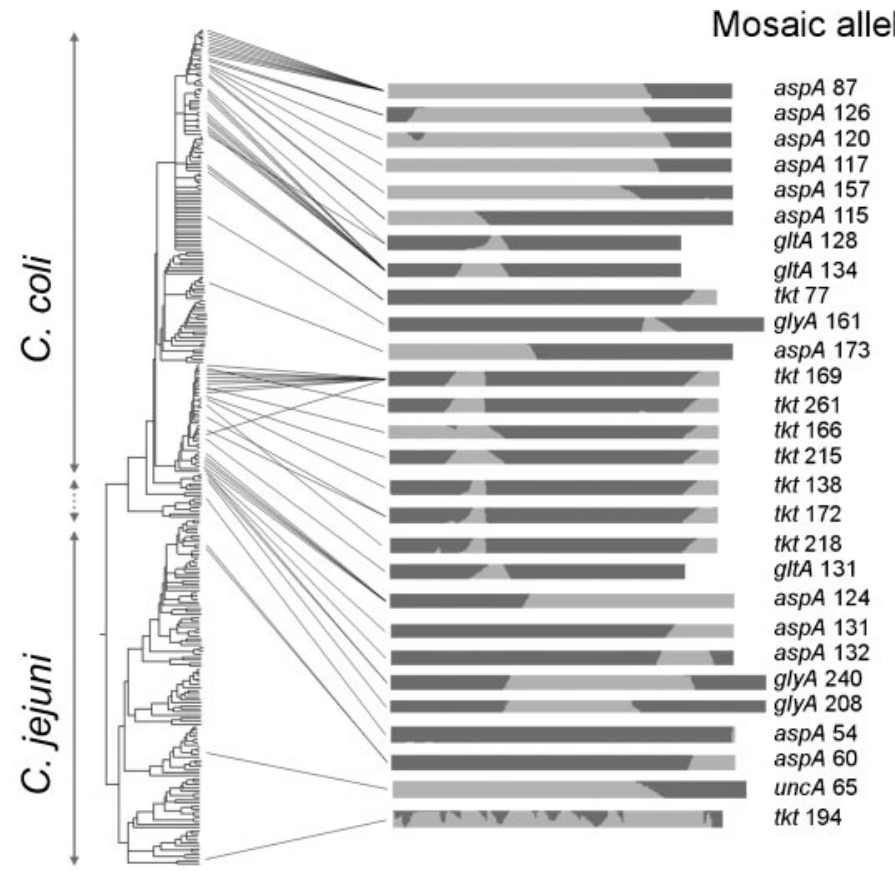

Introductions

C. coli 1

C. coli 1

C. coli 1

C. coli 1

C. coli 1

C. colli 1

C. coll 2

C. coli 2

C. coli 3

C. colli 4

C. coli 5

C. coli 3

C. coli 3

C. coli 3

C. coli 3

C. coli 3

C. coli 3

C. coll 3

C. coli 2

C. coli 6

C. coli 7

C. colli 7

C. coli 8

C. colli 8

C. jejuni 1

C. jejuni 2

C. jejuni 3

C. jejuni 4

(b)

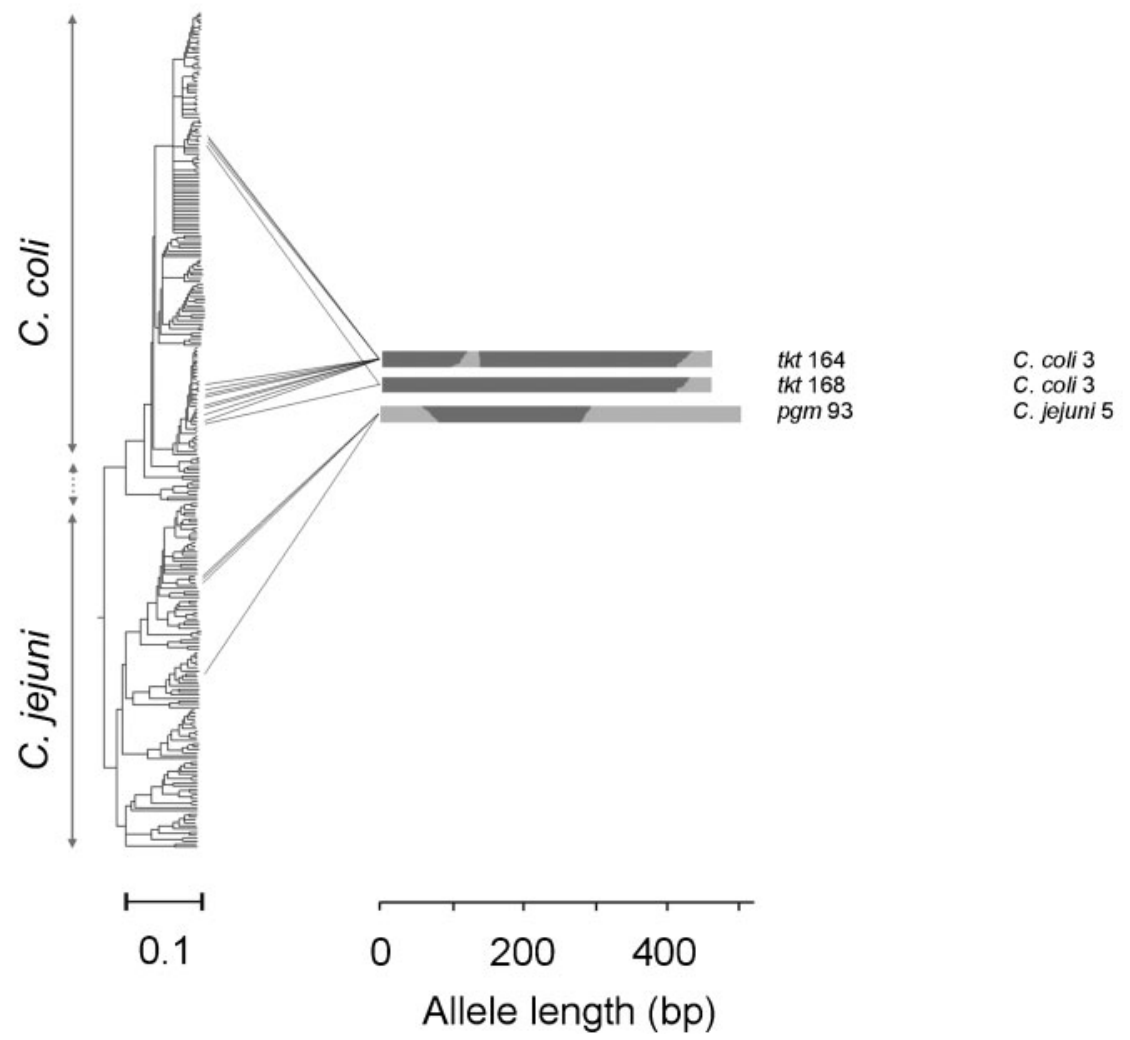

Fig. 2. Distribution of mosaic alleles: consensus trees from CLONALFRAME analysis of concatenated sequences of 275 STs from a combined C. coli and C. jejuni population, including 81 STs containing one or more inter-genomic mosaic alleles. Lines connect the mosaic alleles to the STs in which they occur. Alleles located in (a) closely related and (b) distant clades are indicated in separate trees. Site-by-site nucleotide ancestry, inferred using a linkage model in STRUCTURE, is given for mosaic alleles describing putative origin within C. jejuni (light grey shading) and C. coli (dark grey shading). Background population structure $(k=2)$ was defined using non-recombinant STs. The number of founding introductions was estimated from shared patterns of mosaicism among alleles and the clustering of mosaic alleles on the tree. 
(a)

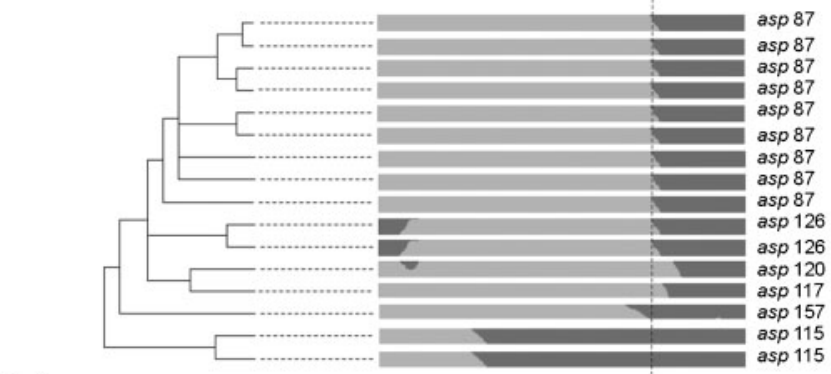

(b)
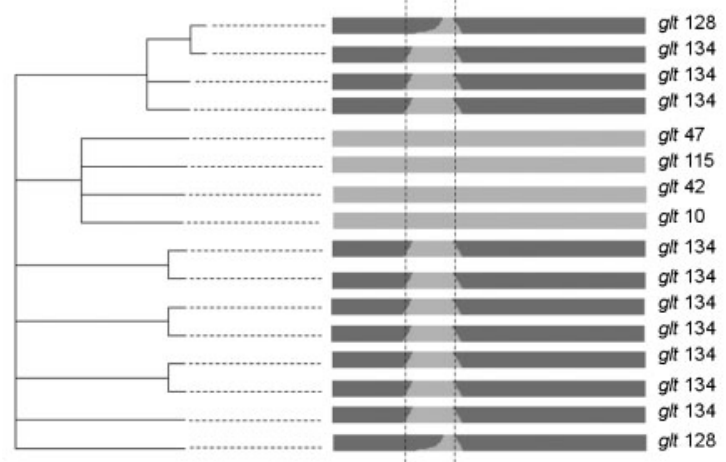

(c)

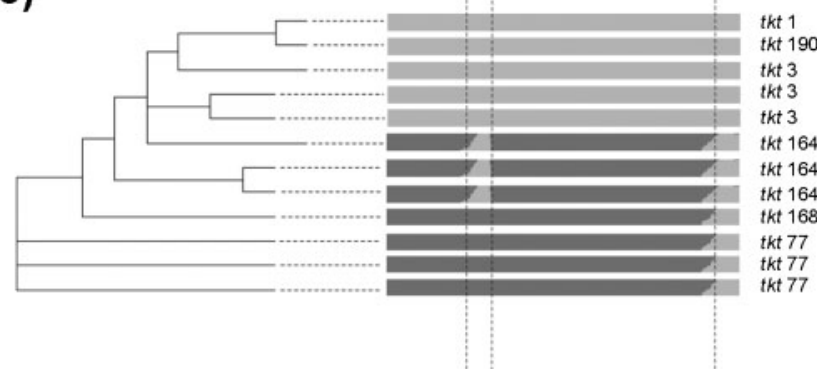

(d)

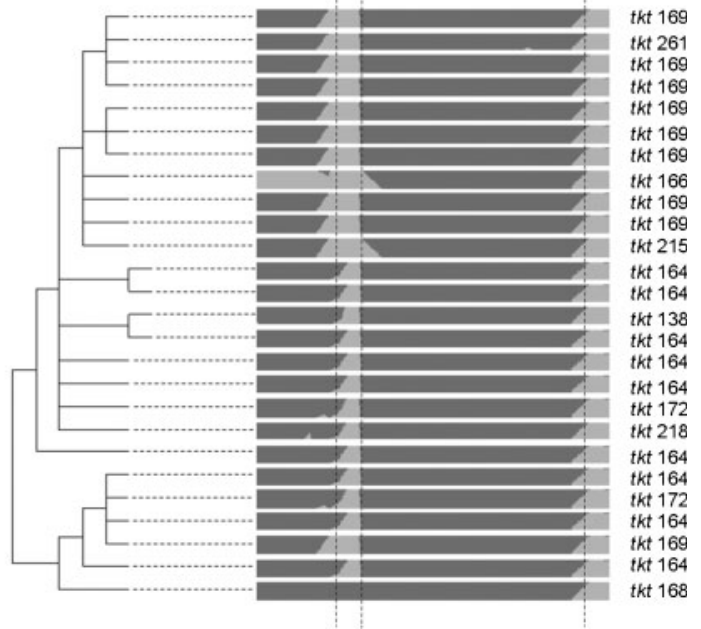

Fig. 3. Major mosaic allele clusters: enlarged CLONALFRAME tree topologies and mosaic allele intra-allelic recombinant regions from the four largest ST clusters containing mosaic alleles of the $\operatorname{asp} A$ (a), gltA (b) and tkt [tkt1 (c) and tkt2 (d)] loci. Site-by-site putative nucleotide ancestry is given for the numbered mosaic alleles (C. jejuni, light grey shading, C. coli, dark grey shading), and the approximate terminal positions of recombinant fragments are indicated with broken lines.

if the whole allele had been replaced would be 5, 46 (aspA); $8,33$ ( $g l t A) ; 14,45$ (tkt1); and $14,45(t k t 2)$. The $n / s$ values for recombinant regions were therefore higher than the average for a whole-allele replacement in the gltA $(0.25>$ $0.24)$, tkt1 $(0.58>0.31)$ and tkt2 $(0.53>0.31)$ clusters. However, although the observed cross-species imports in these examples had a higher proportion of synonymous changes than the part of the allele that was not present, the number of polymorphisms was low, and comparison of much larger sequence tracts would be necessary to confirm if this is because regions with a high proportion of nonsynonymous mutations were not imported or, more probably, if they were eroded to a greater extent by later events. Similarly, while assignment of individual polymorphisms within the mosaic alleles with STRUCTURE indicated that the recombinant regions had a greater mean assignment probability to $C$. coli than the polymorphisms in the eroded regions for the aspA $(0.11>0.1)$, gltA $(0.33>0.08)$, tkt1 $(0.24>0.09)$ and $t k t 2(0.18>0.0 .07)$ mosaic allele clusters, the number of polymorphisms was too low to test if imported C. jejuni DNA that remained in C. coli alleles was more similar to the original C. coli sequence than that which had theoretically been removed.

\section{DISCUSSION}

The patterns of mosaic alleles in C. jejuni and C. coli clade 1 were consistent with a recent increase in gene flow between these two organisms, as suggested by the analysis of wholeallele replacements of housekeeping genes reported previously (Sheppard et al., 2008). As in the whole-allele analysis, which excluded mosaic alleles to ensure a conservative estimate of gene flow, the distribution of mosaic alleles is consistent with frequent introgression of housekeeping genes, or fragments of housekeeping genes, from C. jejuni into C. coli clade 1. The interpretation of the whole MLST allele analysis has been challenged in line with the view that horizontal genetic exchange of housekeeping genes must be infrequent in the absence of strong positive selection or hitchhiking (Caro-Quintero et al., 2009). However, the analysis of Caro-Quintero et al. (2009) did not use a formal population-genetic approach for assigning species and clade designations, and did not identify the clades within C. coli, significantly altering the estimates of gene flow obtained. These alternative estimates were further affected by the use of the catalogue of ST and allele variation present in the PubMLST database as a representative population sample, which it is not. In 
addition, some hybrids that may have expanded clonally were excluded from the analysis, without a reciprocal exclusion of non-hybrids that may have done so, further altering the impact of introgression, by decreasing the relative proportion of hybrid lineages. Finally, ST data that did not conform to the assumption of low genetic exchange among housekeeping genes were dismissed as clerical errors. Although no evidence was presented to substantiate this assertion, this last criticism is met by the analysis of mosaic genes alleles, as such clerical errors are not possible and the most likely explanation of mosaic alleles is that they are the product of inter-species recombination. In conclusion, the data presented here on mosaic genes are supportive of the original analysis, which proposed a recent increase in gene flow between $C$. jejuni and C. coli clade 1 that, if sustained, will lead to progressive convergence or despeciation of $C$. coli clade 1, but not identifiably C. coli clades 2 and 3, with C. jejuni (Sheppard et al., 2008).

The levels of introgression obtained from the analysis of mosaic genes were similar, but not identical, to those obtained from whole MLST allele analysis. Within C. coli clade 1, 105 STs $(17 \%)$ and 25 unique alleles (8\%) contained mosaic regions of C. jejuni ancestry (Table 1 ). The latter value is somewhat lower than the estimate of $18 \%$ introgression based on whole-gene analysis. This is not inconsistent with the earlier findings; however, the MLST data for these organisms suggest that the average recombination fragment size is considerably larger than the average size of the of the sequences used to define MLST alleles (402-507 bp) (Sheppard et al., 2008; Wilson et al., 2009): therefore much of the variation is the result of reassortment of existing alleles (Dingle et al., 2001; Wilson et al., 2009) and analysis of mosaic alleles is likely to underestimate introgression from C. jejuni to C. coli. In addition, the method for defining mosaic alleles ignores alleles where $<25 \%$ of DNA is introgressed and therefore the estimate of $8 \%$ of $C$. coli genes representing introgression events from $C$. jejuni is conservative for this dataset. It is noteworthy that the number of unique introgression events between $C$. jejuni and C. coli clade 1 appears to be similar in both directions (eight from $C$. jejuni to C. coli clade 1; five from C. coli to C. jejuni) so that the differences in the influence of the introgressed DNA in each species is apparently dependent on the fate of the hybrids and mosaics after these events have occurred. Indeed there is only one common example of the persistence of $C$. coli clade 1 DNA in C. jejuni, that of the uncA17 allele in ST-61 clonal complex isolates. This contrasts with the large amounts of $C$. jejuni DNA within $C$. coli clade 1 STs (Sheppard et al., 2008) and alleles reported here.

It is likely that mosaic alleles are continually generated among closely related transformable bacteria, as evidenced by many anecdotal reports of their occurrence (Dingle et al., 2005; Feil et al., 1995; Zhou \& Spratt, 1992; Zhou et al., 1997), but remain at low frequency. In some cases mosaic genes spread within a population as a result of positive selection for a novel adaptive phenotype that they confer. A well-established example of this is the spread of mosaic genes encoding penicillin-binding proteins, which are associated with increased penicillin resistance in a number of bacteria (Coffey et al., 1995; Dowson et al., 1990). For pathogenic and commensal bacteria selection pressures may be different at different stages in transmission. For example, in the species Neisseria meningitidis, bacteria with mosaic antigen-encoding genes generated within hosts by interspecies genetic exchange, apparently experiencing an advantage in that host as a consequence of the host immune response, appear to be less fit in onward human-to-human transmission such that the hybrids are subsequently lost (Zhou et al., 1997). The extent to which stabilizing selection affects the spread of mosaic housekeeping genes remains unclear, but reduced selection against introgressed genes and mosaic genes could explain the observed increase in the proportion of whole-gene replacements and mosaic C. jejuni genes in C. coli clade 1. The patterns of polymorphisms within the mosaics are broadly supportive of a role of some selection against mosaics, but this is based on a small number of polymorphisms, and more data are required to investigate this possibility more rigorously.

The patterns of variation across bacterial genomes in features such as gene order, distribution of coding sequences on leading and lagging strands, GC skew, and codon usage are consistent with selection operating on sequence features other than maintenance of the protein sequences encoded (Bentley \& Parkhill, 2004). A number of studies have indicated pervasive selection pressures across much of the genome in Escherichia coli and Salmonella enterica (Charlesworth \& Eyre-Walker, 2006) and the genus Campylobacter (Lefébure \& Stanhope, 2009). It is possible that intensive agriculture has generated a novel niche in which both $C$. jejuni and C. coli clade 1 thrive, promoting genetic exchange between these two bacteria, with a concomitant change in selective pressure that favours or tolerates mosaic alleles and whole-gene hybrids, but this intriguing possibility requires much more investigation. With whole-genome analyses of multiple isolates becoming increasingly possible, such studies are now feasible, and the interactions of $C$. jejuni and C. coli clade 1 populations at the genomic level provide a model system for distinguishing between the various contrasting theoretical frameworks currently being proposed to explain the relative roles of selection, genetic exchange and neutral mechanisms in the evolution and maintenance of genetic structure in bacterial populations.

\section{ACKNOWLEDGEMENTS}

We thank Daniel Falush for helpful comments. We are grateful to Frances Colles and Alison Cody, who curate the Campylobacter Multi-locus Sequence Typing Website (http://pubmlst.org/ campylobacter/) and all of those who contribute to it. S. K.S. is a Wellcome Trust Career Development Fellow and M.C.J.M. a Wellcome Trust Senior Research Fellow. 


\section{REFERENCES}

Altschul, S. F., Gish, W., Miller, W., Myers, E. W. \& Lipman, D. J. (1990). Basic local alignment search tool. J Mol Biol 215, 403-410.

Baltrus, D. A., Guillemin, K. \& Phillips, P. C. (2008). Natural transformation increases the rate of adaptation in the human pathogen Helicobacter pylori. Evolution 62, 39-49.

Bentley, S. D. \& Parkhill, J. (2004). Comparative genomic structure of prokaryotes. Annu Rev Genet 38, 771-791.

Brückner, R., Nuhn, M., Reichmann, P., Weber, B. \& Hakenbeck, R. (2004). Mosaic genes and mosaic chromosomes-genomic variation in Streptococcus pneumoniae. Int J Med Microbiol 294, 157-168.

Caro-Quintero, A., Rodriguez-Castaño, G. P. \& Konstantinidis, K. T. (2009). Genomic insights into the convergence and pathogenicity factors of Campylobacter jejuni and Campylobacter coli species. J Bacteriol 191, 5824-5831.

Charlesworth, J. \& Eyre-Walker, A. (2006). The rate of adaptive evolution in enteric bacteria. Mol Biol Evol 23, 1348-1356.

Coffey, T. J., Daniels, M., McDougal, L. K., Dowson, C. G., Tenover, F. C. \& Spratt, B. G. (1995). Genetic analysis of clinical isolates of Streptococcus pneumoniae with high-level resistance to expandedspectrum cephalosporins. Antimicrob Agents Chemother 39, 13061313.

Cohan, F. M. \& Koeppel, A. F. (2008). The origins of ecological diversity in prokaryotes. Curr Biol 18, R1024-R1034.

Cooper, T. F. (2007). Recombination speeds adaptation by reducing competition between beneficial mutations in populations of Escherichia coli. PLoS Biol 5, e225.

Didelot, X. \& Falush, D. (2007). Inference of bacterial microevolution using multilocus sequence data. Genetics 175, 1251-1266.

Didelot, X. \& Maiden, M. C. (2010). Impact of recombination on bacterial evolution. Trends Microbiol 18, 315-322.

Dingle, K. E., Colles, F. M., Wareing, D. R. A., Ure, R., Fox, A. J., Bolton, F. E., Bootsma, H. J., Willems, R. J., Urwin, R. \& Maiden, M. C. (2001). Multilocus sequence typing system for Campylobacter jejuni. J Clin Microbiol 39, 14-23.

Dingle, K. E., Colles, F. M., Falush, D. \& Maiden, M. C. (2005). Sequence typing and comparison of population biology of Campylobacter coli and Campylobacter jejuni. J Clin Microbiol 43, 340-347.

Doolittle, W. F. (2008). Microbial evolution: stalking the wild bacterial species. Curr Biol 18, R565-R567.

Dowson, C. G., Hutchison, A., Woodford, N., Johnson, A. P., George, R. C. \& Spratt, B. G. (1990). Penicillin-resistant viridans streptococci have obtained altered penicillin-binding protein genes from penicillin-resistant strains of Streptococcus pneumoniae. Proc Natl Acad Sci U $S$ A 87, 5858-5862.

Excoffier, L., Laval, G. \& Schneider, S. (2005). Arlequin (version 3.0): an integrated software package for population genetics data analysis. Evol Bioinform Online 1, 47-50.

Falush, D., Kraft, C., Taylor, N. S., Correa, P., Fox, J. G., Achtman, M. \& Suerbaum, S. (2001). Recombination and mutation during longterm gastric colonization by Helicobacter pylori: estimates of clock rates, recombination size, and minimal age. Proc Natl Acad Sci U S A 98, 15056-15061.

Falush, D., Stephens, M. \& Pritchard, J. K. (2003). Inference of population structure using multilocus genotype data: linked loci and correlated allele frequencies. Genetics 164, 1567-1587.

Feil, E. J. \& Spratt, B. G. (2001). Recombination and the population structures of bacterial pathogens. Annu Rev Microbiol 55, 561590.
Feil, E., Carpenter, G. \& Spratt, B. G. (1995). Electrophoretic variation in adenylate kinase of Neisseria meningitidis is due to inter- and intraspecies recombination. Proc Natl Acad Sci U S A 92, 10535-10539.

Fraser, C., Hanage, W. P. \& Spratt, B. G. (2007). Recombination and the nature of bacterial speciation. Science 315, 476-480.

Fraser, C., Alm, E. J., Polz, M. F., Spratt, B. G. \& Hanage, W. P. (2009). The bacterial species challenge: making sense of genetic and ecological diversity. Science 323, 741-746.

Friis, C., Wassenaar, T. M., Javed, M. A., Snipen, L., Lagesen, K., Hallin, P. F., Newell, D. G., Toszeghy, M., Ridley, A. \& other authors (2010). Genomic characterization of Campylobacter jejuni strain M1. PLoS ONE 5, e12253.

Gutacker, M. M., Mathema, B., Soini, H., Shashkina, E., Kreiswirth, B. N., Graviss, E. A. \& Musser, J. M. (2006). Single-nucleotide polymorphism-based population genetic analysis of Mycobacterium tuberculosis strains from 4 geographic sites. J Infect Dis 193, 121-128.

Hakenbeck, R. (1998). Mosaic genes and their role in penicillinresistant Streptococcus pneumoniae. Electrophoresis 19, 597-601.

Halter, R., Pohlner, J. \& Meyer, T. F. (1989). Mosaic-like organization of IgA protease genes in Neisseria gonorrhoeae generated by horizontal genetic exchange in vivo. EMBO J 8, 2737-2744.

Hanage, W. P., Fraser, C. \& Spratt, B. G. (2005). Fuzzy species among recombinogenic bacteria. $B M C$ Biol 3, 6 .

Hanage, W. P., Spratt, B. G., Turner, K. M. \& Fraser, C. (2006). Modelling bacterial speciation. Philos Trans R Soc Lond B Biol Sci 361, 2039-2044.

Hollingshead, S. K., Becker, R. \& Briles, D. E. (2000). Diversity of PspA: mosaic genes and evidence for past recombination in Streptococcus pneumoniae. Infect Immun 68, 5889-5900.

Jolley, K. A., Feil, E. J., Chan, M. S. \& Maiden, M. C. (2001). Sequence type analysis and recombinational tests (START). Bioinformatics 17, $1230-1231$.

Jolley, K. A., Chan, M. S. \& Maiden, M. C. (2004). mlstdbNet distributed multi-locus sequence typing (MLST) databases. BMC Bioinformatics 5, 86.

Kapur, V., Kanjilal, S., Hamrick, M. R., Li, L.-L., Whittam, T. S., Sawyer, S. A. \& Musser, J. M. (1995). Molecular population genetic analysis of the streptokinase gene of Streptococcus pyogenes: mosaic alleles generated by recombination. Mol Microbiol 16, 509-519.

Kidgell, C., Reichard, U., Wain, J., Linz, B., Torpdahl, M., Dougan, G. \& Achtman, M. (2002). Salmonella typhi, the causative agent of typhoid fever, is approximately 50,000 years old. Infect Genet Evol 2, 39-45.

Kulick, S., Moccia, C., Didelot, X., Falush, D., Kraft, C. \& Suerbaum, S. (2008). Mosaic DNA imports with interspersions of recipient sequence after natural transformation of Helicobacter pylori. PLoS ONE 3, e3797.

Kumar, S., Tamura, K. \& Nei, M. (2004). MEGA3: integrated software for Molecular Evolutionary Genetics Analysis and sequence alignment. Brief Bioinform 5, 150-163.

Lawrence, J. G. (1999). Gene transfer, speciation, and the evolution of bacterial genomes. Curr Opin Microbiol 2, 519-523.

Lawrence, J. G. (2002). Gene transfer in bacteria: speciation without species? Theor Popul Biol 61, 449-460.

Lederberg, J. \& Tatum, E. L. (1946). Gene recombination in Escherichia coli. Nature 158, 558.

Lefébure, T. \& Stanhope, M. J. (2009). Pervasive, genome-wide positive selection leading to functional divergence in the bacterial genus Campylobacter. Genome Res 19, 1224-1232.

Lorenz, M. G. \& Wackernagel, W. (1994). Bacterial gene transfer by natural genetic transformation in the environment. Microbiol Rev 58, 563-602. 
Maiden, M. C. (2006). Multilocus sequence typing of bacteria. Annu Rev Microbiol 60, 561-588.

Maiden, M. C. J., Malorny, B. \& Achtman, M. (1996). A global gene pool in the Neisseriae. Mol Microbiol 21, 1297-1298.

Maynard Smith, J. (1992). Analyzing the mosaic structure of genes. J Mol Evol 34, 126-129.

Maynard Smith, J., Dowson, C. G. \& Spratt, B. G. (1991). Localized sex in bacteria. Nature 349, 29-31.

Maynard Smith, J., Feil, E. J. \& Smith, N. H. (2000). Population structure and evolutionary dynamics of pathogenic bacteria. Bioessays 22, 1115-1122.

Milkman, R. \& Stoltzfus, A. (1988). Molecular evolution of the Escherichia coli chromosome. II. Clonal segments. Genetics 120, 359366.

Ochman, H. \& Groisman, E. A. (1994). The origin and evolution of species differences in Escherichia coli and Salmonella typhimurium. EXS 69, 479-493.

Ochman, H., Lawrence, J. G. \& Groisman, E. A. (2000). Lateral gene transfer and the nature of bacterial innovation. Nature 405, 299-304.

Pritchard, J. K., Stephens, M. \& Donnelly, P. (2000). Inference of population structure using multilocus genotype data. Genetics 155, 945-959.

Rozas, J., Sánchez-DelBarrio, J. C., Messeguer, X. \& Rozas, R. (2003). DnaSP, DNA polymorphism analyses by the coalescent and other methods. Bioinformatics 19, 2496-2497.

Sheppard, S. K., McCarthy, N. D., Falush, D. \& Maiden, M. C. (2008). Convergence of Campylobacter species: implications for bacterial evolution. Science 320, 237-239.

Sheppard, S. K., Dallas, J. F., Strachan, N. J., MacRae, M., McCarthy, N. D., Wilson, D. J., Gormley, F. J., Falush, D., Ogden, I. D. \& other authors (2009). Campylobacter genotyping to determine the source of human infection. Clin Infect Dis 48, 1072-1078.
Snyder, L. A., McGowan, S., Rogers, M., Duro, E., O'Farrell, E. \& Saunders, N. J. (2007). The repertoire of minimal mobile elements in the Neisseria species and evidence that these are involved in horizontal gene transfer in other bacteria. Mol Biol Evol 24, 28022815.

Spratt, B. G. (1988). Hybrid penicillin-binding proteins in penicillinresistant strains of Neisseria gonorrhoeae. Nature 332, 173-176.

Spratt, B. G., Dowson, C. G., Zhang, Q.-Y., Bowler, L. D., Brannigan, J. A. \& Hutchison, A. (1991). Mosaic genes, hybrid penicillin-binding proteins, and the origins of penicillin resistance in Neisseria meningitidis and Streptococcus pneumoniae. In Perspectives on cellular regulation: from bacteria to cancer, pp. 73-83. Edited by J Campisi \& other authors. New York: Wiley-Liss, Inc.

Spratt, B. G., Hanage, W. P. \& Feil, E. J. (2001). The relative contributions of recombination and point mutation to the diversification of bacterial clones. Curr Opin Microbiol 4, 602-606.

Stoltzfus, A., Leslie, J. F. \& Milkman, R. (1988). Molecular evolution of the Escherichia coli chromosome. I. Analysis of structure and natural variation in a previously uncharacterized region between $\operatorname{tr} p$ and tonB. Genetics 120, 345-358.

Wilson, D. J., Gabriel, E., Leatherbarrow, A. J., Cheesbrough, J., Gee, S., Bolton, E., Fox, A., Hart, C. A., Diggle, P. J. \& Fearnhead, P. (2009). Rapid evolution and the importance of recombination to the gastroenteric pathogen Campylobacter jejuni. Mol Biol Evol 26, 385-397.

Zhou, J. \& Spratt, B. G. (1992). Sequence diversity within the $\arg F, f b p$ and $r e c A$ genes of natural isolates of Neisseria meningitidis: interspecies recombination within the $\operatorname{argF}$ gene. Mol Microbiol 6, 2135-2146.

Zhou, J., Bowler, L. D. \& Spratt, B. G. (1997). Interspecies recombination, and phylogenetic distortions, within the glutamine synthetase and shikimate dehydrogenase genes of Neisseria meningitidis and commensal Neisseria species. Mol Microbiol 23, 799-812.

Edited by: D. W. Ussery 